Review

\title{
Graphene Thermal Properties: Applications in Thermal Management and Energy Storage
}

\author{
Jackie D. Renteria ${ }^{1,2}$, Denis L. Nika ${ }^{1,3}$ and Alexander A. Balandin $1,2, *$ \\ 1 Nano-Device Laboratory (NDL) and Phonon Optimized Engineered Materials (POEM) Center, \\ Department of Electrical Engineering and Materials Science and Engineering Program, \\ University of California—Riverside, Riverside, CA 92521, USA; \\ E-Mails: jrent002@ucr.edu (J.D.R.); dlnika@yahoo.com (D.L.N.) \\ 2 Quantum Seed LLC, 1190 Columbia Avenue, Riverside, CA 92507, USA \\ 3 E. Pokatilov Laboratory of Physics and Engineering of Nanomaterials, Department of Physics and \\ Engineering, Moldova State University, Chisinau MD-2009, Moldova \\ * Author to whom correspondence should be addressed; E-Mail: balandin@ee.ucr.edu; \\ Tel.: +1-951-827-2351.
}

External Editor: Philippe Lambin

Received: 3 March 2014; in revised form: 30 September 2014 / Accepted: 13 November 2014 / Published: 28 November 2014

\begin{abstract}
We review the thermal properties of graphene, few-layer graphene and graphene nanoribbons, and discuss practical applications of graphene in thermal management and energy storage. The first part of the review describes the state-of-the-art in the graphene thermal field focusing on recently reported experimental and theoretical data for heat conduction in graphene and graphene nanoribbons. The effects of the sample size, shape, quality, strain distribution, isotope composition, and point-defect concentration are included in the summary. The second part of the review outlines thermal properties of graphene-enhanced phase change materials used in energy storage. It is shown that the use of liquid-phase-exfoliated graphene as filler material in phase change materials is promising for thermal management of high-power-density battery parks. The reported experimental and modeling results indicate that graphene has the potential to outperform metal nanoparticles, carbon nanotubes, and other carbon allotropes as filler in thermal management materials.
\end{abstract}


Keywords: graphene; thermal conductivity; graphene applications; thermal phase change materials

\section{Introduction and Terminology}

In this paper, we review thermal properties of graphene, few-layer graphene (FLG), and graphene nanoribbons (GNR) and provide an example of a graphene application in thermal phase change materials (PCM). In many cases, when discussing graphene thermal applications, we use the term graphene even when the actual material consists of a mixture of single layer graphene (SLG), bilayer graphene (BLG), and FLG. The latter is because for thermal applications the difference between SLG and FLG is not as important as for electronic applications. It is sometimes difficult to distinguish between FLG and graphite films or between FLG and graphite nano-platelets (GnP) used in composite materials. The definition of SLG - a single atomic plane of $\mathrm{sp}^{2}$-bound carbon-is strict. The distinction between FLG and thin film of graphite or bulk graphite depends on the context. Investigating electrical properties one can consider the material to be FLG rather than graphite as long as it is thin enough for changing its carrier density via the electrostatic gating. In the thermal field, one can consider the film to be FLG as long as its Raman spectrum is different from that of bulk graphite.

Acoustic phonons determine the thermal properties of graphene and graphite at room temperature (RT) $T=300 \mathrm{~K}$, while the optical phonons define their Raman spectrum [1-5]. In both cases, the crystal lattice dynamics is essential for the distinction between FLG and graphite. The Raman spectrum of FLG differs from that of graphite for the thickness $H$ less than seven to 10 atomic planes [6-11]. In this review, we consider the materials to be FLG when its phonon properties are different from those of graphite $(H \leq 10 \approx 3.5 \mathrm{~nm})$. In most of cases, FLG flakes have larger lateral sizes (up to a few micrometers) than their thickness $H$.

The initial interest in the thermal properties of graphene and FLG was driven by the exotic physics of two-dimensional (2D) phonon transport [1-5]. Recently, the studies turned to thermal properties of graphene and related composite materials from the position of practical applications. In this review, we briefly outline the state-of-the-art and new developments in the field of thermal properties of graphene. In addition, as an example of a practical application of graphene in thermal management, we will describe graphene-enhanced phase-change materials (PCMs). Readers interested in details of the phonon thermal transport in graphene and FLG are referred to other recent reviewers $[1-3,12,13]$.

\section{Motivations for Graphene Applications in Thermal Management}

Development of high-power-density batteries, e.g., Li-ion batteries, enabled progress in mobile communications, consumer electronics, and automotive industries [14-16]. Temperature rise beyond the normal operating range negatively affects Li-ion battery performance. If overheated, the battery can suffer thermal runaway, cell rupture or explosion [17-19]. A conventional approach for thermal management of high-power-density-ion battery packs is based on the utilization of thermal PCMs. They reduce the temperature rise in the battery due to the latent heat storing and phase changes over a small temperature range [20-22]. 
The common PCMs have very low thermal conductivity, $K$, with typical values in the range of 0.17-0.35 W/mK at RT [23]. For comparison, the room-temperature (RT) thermal conductivity of Si and $\mathrm{Cu}$ are $\sim 145 \mathrm{~W} / \mathrm{mK}$ and $\sim 381 \mathrm{~W} / \mathrm{mK}$, respectively. Conventional PCMs store heat from the batteries rather than transfer it away from the battery pack. The use of PCM in battery cells also serves the purpose of buffering the battery cell from extreme fluctuations in ambient temperature. This is different from thermal management of computer chips. To reduce the temperature rise in a computer chip, one uses thermal interface materials (TIMs) or heat spreaders that facilitate heat transfer from the chip to the heat sink $[15,24,25]$. The thermal conductivity of TIMs is in the range of $1-10 \mathrm{~W} / \mathrm{mK}$ while that of solid graphite-based heat spreaders can be on the order of $1000 \mathrm{~W} / \mathrm{mK}$ [26]. In this review we describe how these two different approaches for thermal management can be combined via the introduction of the hybrid PCM with graphene acting as filler for increased thermal conductivity. The properties that allow graphene to be an exceptional filler material are its high intrinsic thermal conductivity [1,2], and strong binding with various matrix materials [25,27-30]. The discussion of graphene applications in PCMs will mostly follow our work reported in Ref. [29].

\section{Intrinsic Thermal Conductivity of Graphene}

We start by summarizing the state of the art in the fundamental understanding of the thermal properties of graphene, focusing on recent theoretical and experimental reports. In 2008, it was discovered at the University of California-Riverside that graphene has extremely high intrinsic thermal conductivity $K$, which can exceed that of carbon nanotubes (CNTs) [1-5]. FLG retains excellent thermal properties [1,2,31]. Graphite, which is the 3D bulk limit for FLG with the number of layers $\mathrm{n} \rightarrow \infty$, is still an outstanding heat conductor with the intrinsic $K \approx 2000 \mathrm{~W} / \mathrm{mK}$ at RT. For comparison, $K \approx 430 \mathrm{~W} / \mathrm{mK}$ for silver and is much lower for silver nanoparticles used in TIMs. The first experimental studies of thermal conductivity of graphene were carried out using an original non-contact optothermal technique based on Raman spectroscopy (see Figure 1a,b). Taking into account the temperature shift of Raman G-peak, the temperature profiles for large-area suspended graphene flakes were determined. The thermal conductivity values were extracted from numerical simulations, taking into account temperature profiles and actual size and shape of the flakes. It was established that graphene demonstrates very high thermal conductivity $K$, exceeding $3000 \mathrm{~W} / \mathrm{mK}$ near RT for large graphene flakes [1-5]. The measurements were performed with large-area suspended graphene layers exfoliated from bulk graphite. The development of the optothermal technique was instrumental for carrying out the thermal measurements with graphene.

In the optothermal technique, the heating power, $\Delta P$, is provided with the laser light focused on a suspended graphene layer connected to heat sinks at both ends [1]. The temperature rise, $\Delta T$, in response to the dissipated power, $\Delta P$, is determined with a Raman spectrometer. The Raman $\mathrm{G}$ peak in graphene's spectrum exhibits strong temperature, $\mathrm{T}$, dependence. The calibration of the spectral position of $\mathrm{G}$ peak with temperature has to be performed by changing the sample temperature while using low laser power to avoid local heating. During the thermal conductivity measurements the suspended graphene layer is heated by the increasing laser power. The local temperature rise in graphene is measured as $\Delta T=\Delta \omega_{G} / \varphi_{G}$, where $\varphi_{G}$ is the temperature coefficient of the Raman G peak in the relevant temperature range [1]. 
Figure 1. (a) Schematic of the suspended graphene structure used for measurements of the thermal conductivity of graphene. Graphene was heated with the laser light focused in the middle of the suspended part. The temperature rise was determined from the shift of the $G$ peak position in graphene Raman spectrum; (b) Scanning electron image of the bilayer graphene ribbon suspended across the $3-\mu \mathrm{m}$ trench in $\mathrm{Si} / \mathrm{SiO}_{2}$ wafer for optothermal measurements. The image is reproduced with permission from [1]. Copyright 2010 Nature Publishing Group.
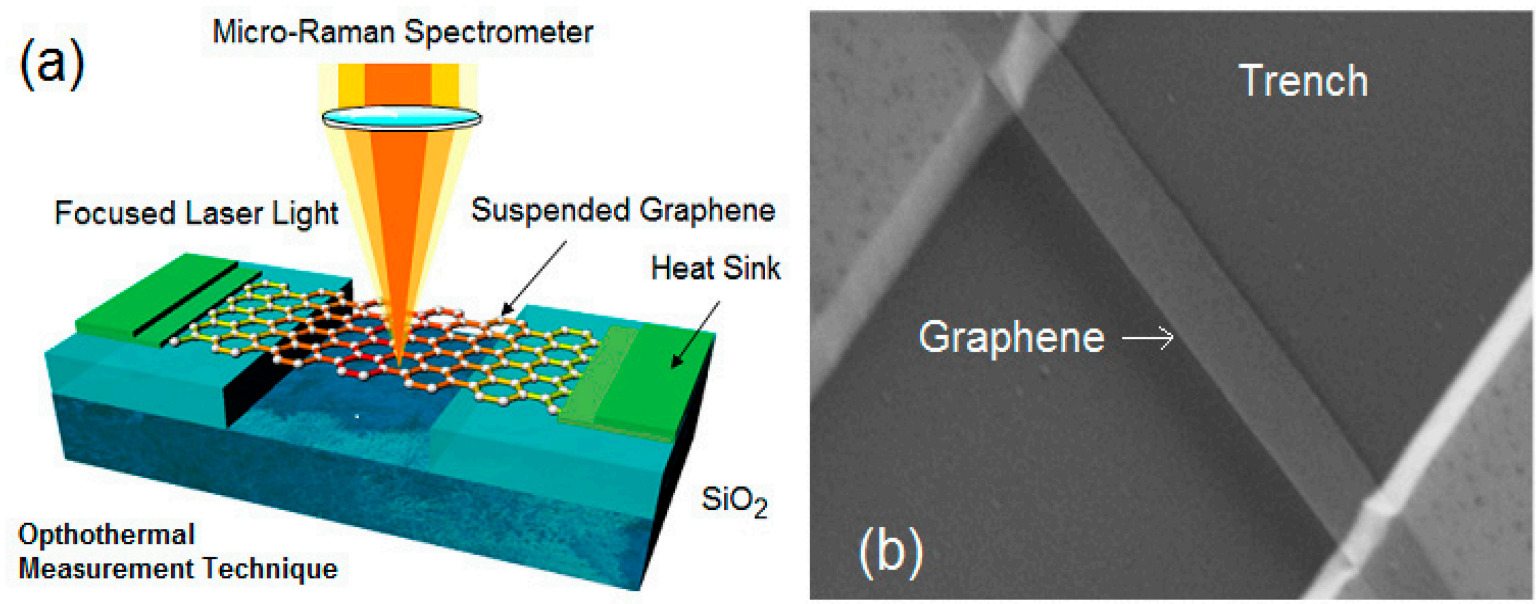

The heat dissipated in graphene can be determined by measuring the integrated Raman intensity of $\mathrm{G}$ peak $[4,5]$ or by a detector placed under the graphene $[32,33]$. Due to the fact that the optical absorption in graphene depends on the wavelength of the light [1,2,34-36], and because it is affected by strain, defects, and multiple reflections for graphene suspended over the trenches, one should measure the optical absorption in the specific experimental conditions. The dependence of the light absorption in graphene on the wavelength results from the many-body effects [34-36]. A correlation between $\Delta T$ and $\Delta P$ for graphene samples with a given geometry gives the thermal conductivity value via solution of the heat diffusion equation. The suspended portion of graphene is needed for determining $\Delta P$, forming a two-dimensional (2-D) heat front propagating toward the heat sinks, and reducing thermal coupling to the substrate. The thermal conductivity data in the Raman optothermal technique can be extracted for the graphene and FLG flakes of arbitrary shape via solution of the heat-diffusion equation using the finite element method [31].

Independent studies conducted by other research groups [32,33] also utilized the non-contact optothermal technique but modified it via addition of a power meter under the suspended portion of the graphene samples. It was found that thermal conductivity of the suspended high-quality CVD graphene exceeded $2500 \mathrm{~W} / \mathrm{mK}$ at $350 \mathrm{~K}$, and it was as high as $K \approx 1400 \mathrm{~W} / \mathrm{mK}$ at $500 \mathrm{~K}$ [32]. The reported value is larger than thermal conductivity of bulk graphite at RT, which is about $2000 \mathrm{~W} / \mathrm{mK}$. Other optothermal studies with suspended graphene found thermal conductivity in the range from 1500 to $5000 \mathrm{~W} / \mathrm{mK}$ [33]. The experimental data for suspended or partially suspended graphene is closer to the intrinsic thermal conductivity because suspension reduces thermal coupling to the substrate and scattering on the substrate defects and impurities. The measurements for exfoliated graphene supported on $\mathrm{SiO}_{2} / \mathrm{Si}$ substrate revealed in-plane $K \approx 600 \mathrm{~W} / \mathrm{mK}$ near RT [37]. This value is below those reported for suspended graphene because of the phonon scattering at the interface and 
diffusion to the substrate. The thermal conductivity of supported graphene, limited by the extrinsic effects, it is still very high and it exceeds that of silicon and copper. In Ref. [37], the authors calculated the theoretical thermal conductivity of free graphene to be $\sim 3000 \mathrm{~W} / \mathrm{mK}$ near RT. Detailed review of thermal conductivity measurements and comparison of the results among different groups was given in Ref. [1].

\section{Theory of the Thermal Conductivity of Graphene and GNR}

The first experimental investigations of the thermal properties in graphene materials [4,5,31-33,37] stimulated numerous theoretical and computational works in the field (see Figure 2a,b). Here, we briefly review the state-of-the-art in theory of thermal transport in graphene and GNRs. Many different theoretical models have been proposed for the prediction of the phonon and thermal properties in graphite, graphene and GNRs during the last few years. The phonon energy spectra have been theoretically investigated using Perdew-Burke-Ernzerhof generalized gradient approximation (GGA) [38-40], valence-force-field (VFF) and Born-von Karman models of lattice vibrations [41-46], continuum approach [47-49], first-order local density approximation [39,50,51], fifth- and fourth-nearest neighbor force constant approaches [40,52] or utilized the Tersoff, Brenner or Lennard-Jones potentials [53-55]. The thermal conductivity investigations have been performed within molecular dynamics simulations [56-72], density functional theory [73,74], Green's function method [75,76] and Boltzmann-transport-equation (BTE) approach [31,41-43,49,53-55,77-85]. It has been shown that phonon energies strongly depend on the interatomic force constants (IFCs) - fitting parameters of interatomic interactions, used in the majority of the models. Therefore a proper choice of interatomic force constants is crucial for the accurate description of phonon energy spectra and thermal conductivity in graphene, twisted graphene and graphene nanoribbons $[1-3,44,86]$.

Although various models predicted different values of thermal conductivity, they demonstrated consistent results on the strong dependence of graphene lattice thermal conductivity on the extrinsic parameters of flakes: edge quality, FLG thickness, lateral size and shape, lattice strain, isotope, impurity and grain concentration. The molecular dynamic (MD) simulations usually give smaller values of thermal conductivity in comparison with the BTE model and experimental data due to exclusion of long wavelength phonons from the model by a finite size of the simulation domain [2]. The effect of the edge roughness on the thermal conductivity in graphene and GNRs has been investigated in Refs. [41-43,49,57,79-82,84,85,87,88]. The rough edge can suppress the thermal conductivity by an order of magnitude as compared to that in graphene or GNRs with perfect edges due to the boundary scattering of phonons. Impurities, single vacancies, double vacancies and StoneWales defects decrease the thermal conductivity of graphene and GNRs by more than $50 \%-80 \%$ in dependence of the defect concentration [41-43,63-67,79].

A study of thermal conductivity of graphene and GNRs under strain was performed in Refs. [61,73,74,76,89]. An enhancement of the thermal conductivity of up to $36 \%$ for the strained 5-nm armchair or zigzag GNRs was found in the ballistic transport regime [76]. In the diffusive transport regime, the applied strain enhanced the Umklapp scattering and thermal conductivity diminishes by $\sim 1.4$ orders of magnitude at RT in comparison with the unstrained graphene [74]. The authors of Ref. [61] have found that when the strain is applied in both directions - parallel and 
perpendicular to the heat transfer path - the graphene sheets undergo complex reconstructions. As a result, some of the strained graphene structures can have higher thermal conductivity than that of SLG without strain [61]. The discrepancy between theoretical findings and experiments requires additional investigations of thermal transport in strained graphene and GNRs. The isotope composition is another key parameter for thermal conductivity engineering in these materials [1-3,83,90-95]. Naturally occurring carbon materials are made up of two stable isotopes of $12 \mathrm{C}(\sim 99 \%)$ and $13 \mathrm{C}$ $(\sim 1 \%)$. The change in the isotope composition significantly influences the crystal lattice properties. Increasing the "isotope doping" leads to a suppression of the thermal conductivity in graphene and GNRs of up to two orders of magnitude at RT due to the enhanced phonon-point defect (mass-difference) scattering [83,90-95].

Figure 2. (a) Dependence of the thermal conductivity of the rectangular graphene ribbon on the ribbon length $L$ shown for different specular parameters $p$. The ribbon width is fixed at $d=5 \mu \mathrm{m}$; (b) Dependence of the thermal conductivity of the rectangular graphene ribbon on the ribbon length $L$ shown for different ribbon width $d$. The specular parameter is fixed at $p=0.9$. Note in both panels an unusual non-monotonic length dependence of the thermal conductivity, which results from the exceptionally long phonon mean free path of the low-energy phonons and their angle-dependent scattering from the ribbon edge. Reprinted with permission from [43]. Copyright 2012 American Chemical Society.
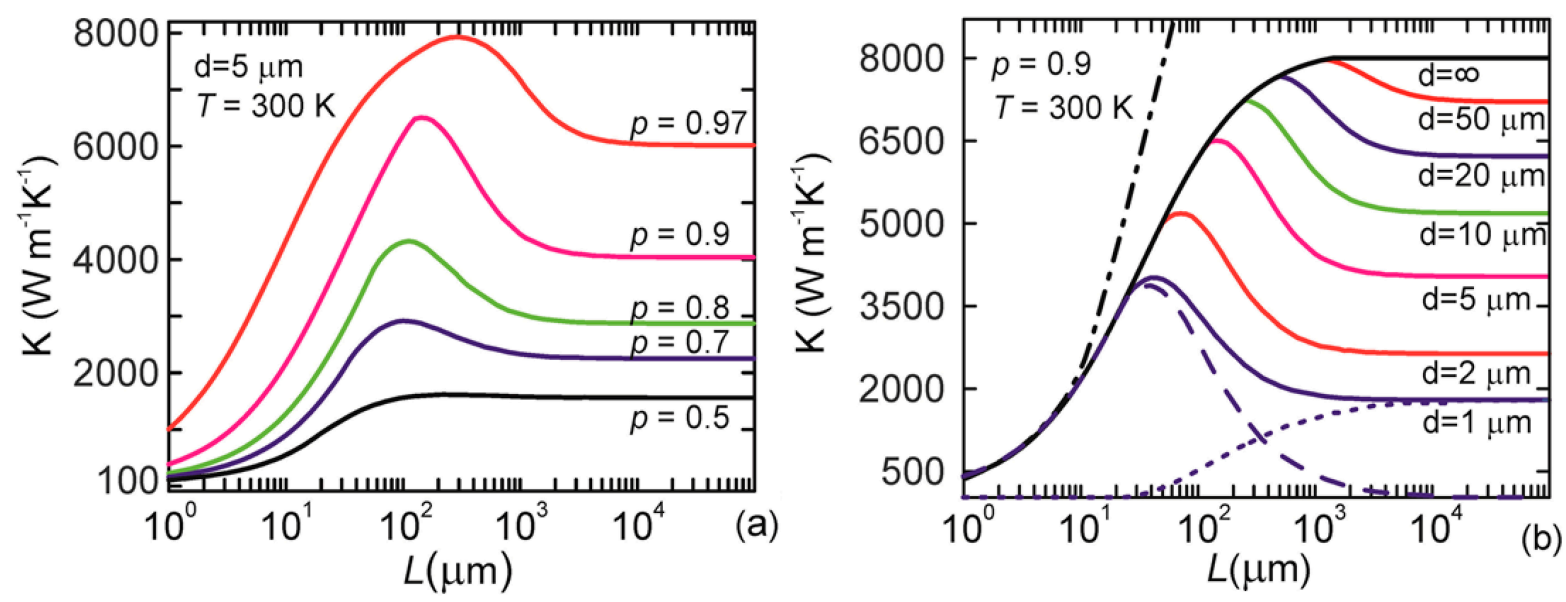

Partial contribution of $L A, T A$ and $Z A$ phonons to the thermal conductivity in graphene is a subject of numerous debates. Some researchers argued that $L A$ and $T A$ phonons carry most of the heat in suspended graphene [41-43,49,72,84,85], while others suggested that $Z A$ modes are dominate heat carriers $[37,54,55,60,96]$. Supporting the graphene by substrates or ripples in suspended graphene flakes increases the scattering of $Z A$ modes and decreases their partial contribution to the thermal conductivity $[37,97]$.

Graphene and GNRs also demonstrated an intriguing dependence of the thermal conductivity on their geometrical parameters: lateral sizes and shapes [41-43,49,68-71,85]. Using the BTE approach, Nika et al. [43] have demonstrated that RT thermal conductivity of a rectangular graphene flake with $5 \mu \mathrm{m}$ width increases with length $L$ up to $L \sim 40-200 \mu \mathrm{m}$ and converges for $L>50-1000 \mu \mathrm{m}$ in dependence on the phonon boundary scattering parameter $p$ (see Figure $2 \mathrm{a}$ ). The dependence of the thermal conductivity on $L$ is non-monotonic, which is explained by the interplay between contribution 
to the thermal conductivity from two groups of phonons: participating and non-participating in the edge scattering [43]. The exceptionally large mean free path (MFP) of the acoustic phonons in graphene is essential for this effect. The increase in the flake width or phonon edge scattering (see Figure 2a,b) attenuates the non-monotonic behavior. It disappears in circular flakes or flakes with very rough edges (with specular parameter $p<0.5$ ). Strong dependence of thermal conductivity on the flake length was recently confirmed experimentally. $\mathrm{Xu}$ et al. [98] reported on the logarithmically divergent room temperature thermal conductivity in suspended graphene flakes with $L \sim 0.01-10 \mu \mathrm{m}$ [98].

A number of studies [68-70] employed the MD simulations to investigate the length dependence of the thermal conductivity in graphene and GNRs. The converged thermal conductivity in graphene was found for $L>16 \mu \mathrm{m}$ in Ref. [68]. In Refs. [69,70], the thermal conductivity increases monotonically with an increase of the length up to $2.8 \mu \mathrm{m}$ in graphene [70] and $800 \mathrm{~nm}$ in GNRs [69]. The obvious length dependence in graphene and GNRs can be attributed to the extremely large phonon mean free path $\Lambda \sim 775 \mathrm{~nm}$ [5], which provides noticeable length dependence even for flakes with micrometer lengths. The available values of phonon thermal conductivity in SLG, few-layer graphene and GNRs are presented in Table 1 at RT (if not indicated otherwise). Readers interested in a more detailed description of theoretical models for the heat conduction in graphene materials are referred to other reviews $[2,13]$.

Table 1. Thermal conductivity of graphene and graphene nanoribbons.

\begin{tabular}{|c|c|c|c|c|}
\hline Sample & $K(\mathbf{W} / \mathbf{m K})$ & Method & Description & Refs. \\
\hline \multicolumn{5}{|c|}{$\begin{array}{r}\text { Experimental Data } \\
\end{array}$} \\
\hline \multirow{6}{*}{ SLG } & $\sim 3000-5000$ & Raman optothermal & Suspended; exfoliated & {$[4,5]$} \\
\hline & 2500 & Raman optothermal & $\begin{array}{l}\text { Suspended; chemical vapor deposition } \\
\text { (CVD) grown }\end{array}$ & {$[32]$} \\
\hline & $1500-5000$ & Raman optothermal & Suspended; CVD grown & {$[33]$} \\
\hline & 600 & Raman optothermal & Suspended; exfoliated; $\mathrm{T} \sim 660 \mathrm{~K}$ & [99] \\
\hline & 600 & Electrical & Supported; exfoliated & {$[37]$} \\
\hline & $310-530$ & $\begin{array}{l}\text { Electrical } \\
\text { self-heating }\end{array}$ & $\begin{array}{l}\text { Exfoliated and chemical vapor deposition grown; } \\
T \sim 1000 \mathrm{~K}\end{array}$ & {$[100]$} \\
\hline \multirow{4}{*}{ FLG } & $1300-2800$ & Raman optothermal & Suspended; exfoliated; $n=2-4$ & [31] \\
\hline & $50-970$ & Heat-spreader method & FLG, encased within $\mathrm{SiO}_{2} ; n=2, \ldots, 21$ & {$[101]$} \\
\hline & $150-1200$ & Electrical self-heating & $\begin{array}{l}\text { Suspended and supported FLG; polymeric residues } \\
\text { on the surface }\end{array}$ & {$[102]$} \\
\hline & $302-596$ & Modified T-bridge & Suspended; $n=2-8$ & [103] \\
\hline $\begin{array}{l}\text { Bilayer } \\
\text { graphene }\end{array}$ & $560-620$ & Electrical self-heating & Suspended; polymeric residues on the surface & [104] \\
\hline \multirow{2}{*}{$\begin{array}{l}\text { FLG } \\
\text { nanoribbons }\end{array}$} & 1100 & Electrical self-heating & Supported; exfoliated; $n<5$ & [105] \\
\hline & $80-150$ & Electrical self-heating & Supported & {$[106]$} \\
\hline
\end{tabular}


Table 1. Cont.

\begin{tabular}{|c|c|c|c|c|}
\hline Sample & $K(\mathbf{W} / \mathbf{m K})$ & Method & Description & Refs. \\
\hline \multicolumn{5}{|c|}{ Theoretical Data } \\
\hline \multirow{15}{*}{ SLG } & $1000-8000$ & BTE, $\gamma_{\mathrm{LA}}, \gamma_{\mathrm{TA}}$ & Strong size dependence & [42] \\
\hline & $2000-8000$ & BTE, $\gamma_{\mathrm{s}}(q)$ & Strong edge, width and grunaisen parameter dependence & [41] \\
\hline & $\sim 2430$ & $\begin{array}{l}\text { BTE, 3rd-order } \\
\text { interatomic force } \\
\text { constants (IFCs) }\end{array}$ & $K$ (graphene $) \geq K($ carbon nanotube $)$ & [107] \\
\hline & $1500-3500$ & BTE, 3rd-order IFCs & Strong size dependence & [54] \\
\hline & $100-8000$ & BTE & Strong length, size, shape and edge dependence & [43] \\
\hline & $2000-4000$ & $\begin{array}{l}\text { Continuum approach } \\
+ \text { BTE }\end{array}$ & Strong isotope, point-defects and strain influence & {$[49,95]$} \\
\hline & 4000 & Ballistic & Strong width dependence & [108] \\
\hline & 2900 & MD simulation & Strong dependence on the vacancy concentration & {$[58]$} \\
\hline & 20000 & $\begin{array}{l}\mathrm{VFF}+\mathrm{MD} \\
\text { simulation }\end{array}$ & $\begin{array}{l}\text { Ballistic regime; flake length } \sim 5 \mu \mathrm{m} \text {; strong width } \\
\text { and length dependence }\end{array}$ & [109] \\
\hline & $100-550$ & MD simulation & $\begin{array}{l}\text { Flake length } L<200 \mathrm{~nm} \text {; strong length and defect } \\
\text { dependence }\end{array}$ & [65] \\
\hline & 3000 & MD simulation & Sheet length $\sim 15 \mu \mathrm{m}$; strong size dependence & [68] \\
\hline & 2360 & MD simulation & $L \sim 5 \mu \mathrm{m}$; strong length dependence & [70] \\
\hline & $4000-6000$ & MD simulation & Strong strain dependence & [74] \\
\hline & 1800 & MD simulation & $6 \mathrm{~nm} \times 6 \mathrm{~nm}$ sheet; isolated & [72] \\
\hline & $1000-1300$ & MD simulation & $\begin{array}{l}6 \mathrm{~nm} \times 6 \mathrm{~nm} \text { sheet; } \mathrm{Cu} \text {-supported; strong } \\
\text { dependence on the interaction strength between } \\
\text { graphene and substrate }\end{array}$ & \\
\hline \multirow{4}{*}{ FLG } & $1000-4000$ & BTE, $\gamma_{\mathrm{s}}(q)$ & $n=8-1$, strong size dependence & [31] \\
\hline & $1000-3500$ & BTE, 3rd-order IFCs & $n=5-1$, strong size dependence & [54] \\
\hline & $2000-3300$ & BTE, 3rd-order IFCs & $n=4-1$ & [55] \\
\hline & $580-880$ & MD simulation & $\begin{array}{l}n=5-1 \text {, strong dependence on the Van-der Vaals } \\
\text { bond strength }\end{array}$ & [59] \\
\hline \multirow{6}{*}{ GNRs } & $1000-7000$ & $\begin{array}{l}\text { Theory: molecular } \\
\text { dynamics, Tersoff }\end{array}$ & Strong ribbon width and edge dependence & [57] \\
\hline & 5500 & BTE & $\begin{array}{l}\text { GNR with width of } 5 \mu \mathrm{m} \text {; strong dependence on the } \\
\text { edge roughness }\end{array}$ & [84] \\
\hline & 2000 & MD simulation & $\begin{array}{l}T=400 \mathrm{~K} ; 1.5 \mathrm{~nm} \times 5.7 \mathrm{~nm} \text { zigzag GNR; strong } \\
\text { edge chirality influence }\end{array}$ & [88] \\
\hline & $30-80$ & $\begin{array}{l}\text { AIREBO potential + } \\
\text { MD simulation }\end{array}$ & $\begin{array}{l}\text { 10-zigzag and 19-arm-chair nanoribbons; strong } \\
\text { defect dependence }\end{array}$ & {$[64,66]$} \\
\hline & $3200-5200$ & MD simulation & $\begin{array}{l}\text { Strong GNRs width }(W) \text { and length dependence; } \\
9 \mathrm{~nm} \leq L \leq 27 \mathrm{~nm} \text { and } 4 \mathrm{~nm} \leq W \leq 18 \mathrm{~nm}\end{array}$ & [67] \\
\hline & $400-600$ & MD simulation & $K \sim L^{0.24} ; 100 \mathrm{~nm} \leq L \leq 650 \mathrm{~nm}$ & [69] \\
\hline $\begin{array}{l}\text { GNRs supported } \\
\text { on } \mathrm{SiO}_{2}\end{array}$ & $100-1000$ & BTE & Strong edge and width dependence & [85] \\
\hline $\begin{array}{l}\text { Few-layer } \\
\text { GNRs }\end{array}$ & $500-300$ & MD simulation & $10-\mathrm{ZGNR}, n=1, \ldots, 5$ & [71] \\
\hline
\end{tabular}




\section{Graphene Applications in Thermal Phase-Change Materials}

In this section we briefly outline the approach of Balandin and co-workers [29] for graphene's practical applications in thermal PCMs and outline results of other groups that reported the use of graphitic nanoparticles for such applications. As an example material system for the composite matrix we selected a specific paraffin wax. Paraffins or paraffinic hydrocarbons have the general composition of $\mathrm{C}_{n} \mathrm{H}_{2 n+2}$ and are straight-chain or branching saturated organic compounds [110]. Commonly used in PCMs, paraffin waxes have the advantages of low cost and availability, are chemically stable, and are durable to cycling. Paraffins are suitable for the thermal control of batteries with its large range of melting points and a high latent heat of fusion $(\sim 250 \mathrm{~kJ} / \mathrm{kg})$. The specific paraffin (IGI-1260) used has a relatively high melting point of $T_{M \sim 70}{ }^{\circ} \mathrm{C}$. The $n$-alkanes distribution of this paraffin wax predominately consists of $\mathrm{C}_{34}-\mathrm{C}_{35}$ hydrocarbons [110]. The paraffin wax is a solid at room temperature and will start to exhibit softening characteristics at elevated temperatures as the long hydrocarbon chains are broken down into smaller ones with the absorption of heat.

As previously reported by some of us [29] the graphene-PCM composites were prepared by melting paraffin wax at $70{ }^{\circ} \mathrm{C}$ on a hot plate and performing high-shear mixing with solutions of graphene and FLG synthesized from industry standard liquid-phase exfoliated (LPE) techniques. To avoid the oxidation process of liquid paraffin waxes, resulting in the formation of peroxide radicals and hydroperoxide above $80{ }^{\circ} \mathrm{C}$, processing temperatures were carefully controlled. The resulting graphene-PCM was poured into molds and allowed to solidify under controlled humidity conditions. Different LPE recipes were used to synthesize the three types of LPE graphene solutions used as filler material. These graphene solutions had different average thicknesses and lateral dimensions. For low loading fractions up to $1 \%$, a graphene solution with an average thickness of one monolayer $(0.35 \mathrm{~nm})$ and a lateral size distribution with a range from 150 to $3000 \mathrm{~nm}$ with $550 \mathrm{~nm}$ average size was used (graphene filler type A). For high loading fractions up to $20 \%$, other types of FLG have been used. Filler type B consisted of a graphene solution with an average thickness of approximately three atomic layers $(\sim 1 \mathrm{~nm})$ and an average lateral dimension of $\sim 10 \mu \mathrm{m}$. Filler type $\mathrm{C}$ had an average flake thickness of $8 \mathrm{~nm}$ with a lateral size in the range of 150 and $3000 \mathrm{~nm}$. Figure 3a presents optical images of hybrid graphene-PCM composites with graphene-FLG loading fractions of 0.5 to 20 wt. \%. These composites were molded into disks and clearly show changes in color from white to black with the addition of graphene. This addition of graphene was also observed to cause some reduction of $T_{M}$. The uniform dispersion and physical coupling of graphene-FLG within the paraffin matrix were verified using scanning electron microscopy (SEM) (see Figure 3b). As confirmed from the SEM images, graphene-FLG fillers are evenly distributed within the matrix. These composites with increasing amounts of graphene were further analyzed and monitored using Raman spectroscopy. Paraffin is known to have a large number of informative bands in its vibrational spectra from which variations are indicative of changes in a paraffin's state and composition [111-115]. Analysis of the observed changes in the Raman spectrum indicated good attachment of graphene to hydrocarbon chains [29]. Additional confirmation of the increasing concentration of $\mathrm{sp}^{2}$-bonded carbon (i.e., graphene filler) and changes to the paraffin's hydrocarbon chain were obtained from X-ray photoelectron spectroscopy (XPS) analysis. Such data provides information relating to the elemental composition, empirical formula, and chemical state of the measured sample. Here, the XPS results 
indicate the presence of hydrocarbons with a binding energy (BE) of $284.9 \mathrm{eV}$. The transition centred at $284.9 \mathrm{eV}$ is characteristic of this paraffin's $\mathrm{C}_{1 \text { s }}$ spectrum and corresponds to the $\mathrm{H}-\mathrm{C}$ and $\mathrm{C}-\mathrm{C}$ bonds. As the relative composition of graphene increases in the hybrid graphene-PCM composite the intensity of the $\mathrm{C}_{1 \text { s }}$ peak changes. The XPS spectra indicate that as more graphene is added and mixed in with the paraffin wax the $\mathrm{sp}^{1}$ hydrocarbon quantity decreases. The reduction in the number of these $\mathrm{H}-\mathrm{C}$ chains in the hybrid composites suggests a reaction is occurring between the graphene and the alkane chains of the paraffin in the hybrid-PCM composite.

Figure 3. Hybrid graphene - paraffin phase change material. (a) Optical image of the PCM samples showing the change in color from white to black with increasing graphene content; (b) Scanning electron microscopy image of the hybrid graphene-PCM indicating uniform distribution of the graphene flakes.

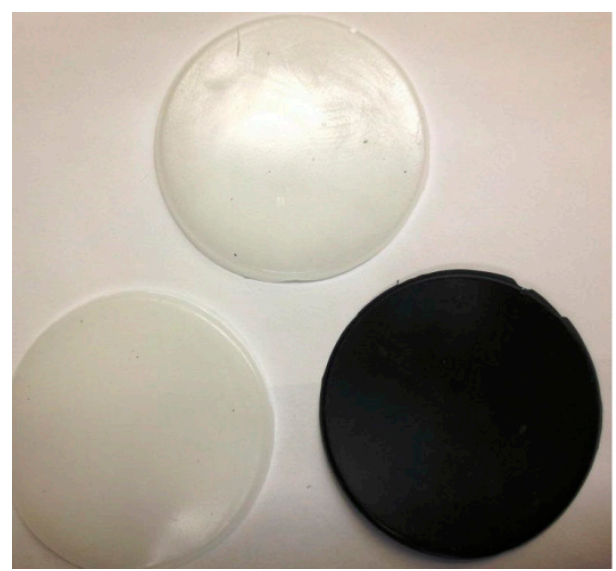

(a)

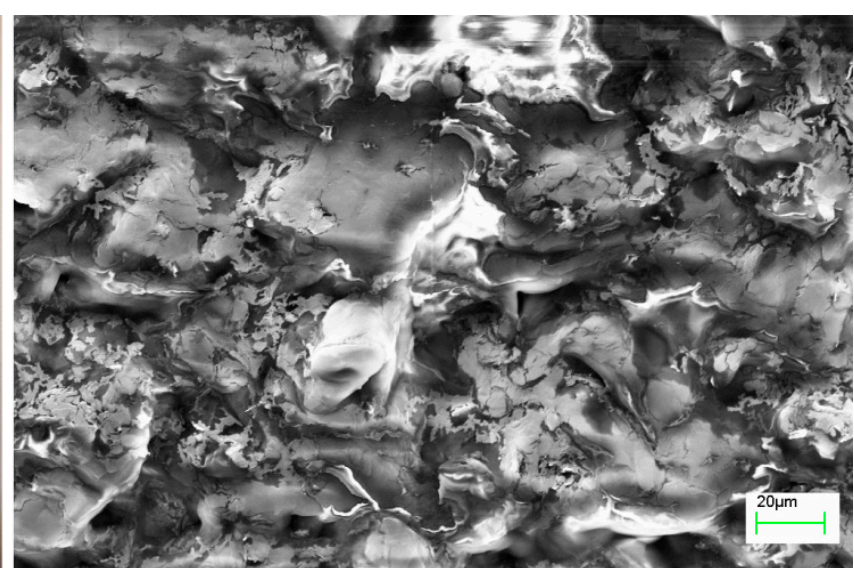

(b)

There are other examples of the use of graphitic nanoparticles for enhancement of paraffin waxes. Additions of nanoparticles to PCMs have been used for temperature regulation of property contrasts between the liquid and solid states. In a specific hexadecane-graphite suspension, a sharp rise in thermal conductivity was observed as the host hexadecane solidified at its transition temperature of $18{ }^{\circ} \mathrm{C}$ [116]. The change in thermal conductivity by a factor of about three near the transition temperature was achieved with a $0.8 \%$ volume fraction of exfoliated graphite flakes processed through sulfuric acid intercalation, microwave expansion, and ultrasonic dispersion [116]. The addition of nanoparticles to PCMs not only aims to improve heat transfer during the melt phase but has also shown to enhance thermal energy storage and solidification of paraffin-based PCMs as with graphite nanofibers (GNFs) in $n$-tricosane [117]. Such GNFs were grown by catalytic deposition of hydrocarbons and carbon monoxide on an iron catalyst in a tube furnace. The resulting composites with 10 wt. \% of GNF provided for a solidification time reduction by $61 \%$ over paraffin samples, demonstrating its benefits to pulsed power profile systems. Another composite of $1 \mathrm{vol} \%$ multilayer graphene suspended in hexadecane demonstrated a solid-liquid thermal conductivity contrast ratio range between $\sim 2$ and 3.0 by freezing rate control [118]. In all aforementioned composites for practical applications and their regulating properties, the thermal transport mechanism occurred through both the nanoparticles and host medium at both liquid and solid host phases. Several instances demonstrate that the adjustment of particulate concentration of the graphite nanoparticles and combinations with a 
variety of waxes provide for novel composites with tunable properties such as thermal and electrical conductivities.

\section{Thermal Conductivity of Graphene-Enhanced Phase Change Materials}

The transient plane source (TPS) method was used to measure the thermal conductivity of the hybrid composites [119-126]. The results of the measurements were compared to those obtained by other experimental techniques [123-124]. Starting with pristine paraffin as the baseline composite, thermal conductivity was measured at $K=0.25 \mathrm{~W} / \mathrm{mK}$. One can see in Figure 4a that as graphene-FLG filler is added to the baseline composite, a drastic increase of $K$ is observed. The hybrid graphene-PCM composite with a $1 \mathrm{wt} . \%$ loading fraction possesses a measured thermal conductivity of $\sim 15 \mathrm{~W} / \mathrm{mK}$ at RT. This represents a significant increase in thermal conductivity by a factor of 60 . This thermal conductivity enhancement factor, defined by $\varepsilon=\left(K-K_{m}\right) / K_{m}$, where $K$ is the measured thermal conductivity of the composite and $K_{m}$ is the thermal conductivity of the paraffin matrix, is exceptionally high compared with values reported for either PCMs with fillers [127-129] or TIMs $[26,28,30]$. The trend continues up to a graphene loading fraction of $20 \mathrm{wt} \%$ where the composite yields the highest value of thermal conductivity at $\sim 45 \mathrm{~W} / \mathrm{mK}$, an enhancement of more than two orders of magnitude. Figure 4a presents the enhancement factors of thermal conductivity among the pristine paraffin wax and hybrid graphene-PCM composites with different graphene-FLG loading fractions [29].

There are several possible reasons for a strong increase in the thermal conductivity of these composites. The first is the arrangement of graphene flakes creating a network within the composite ideal for thermal percolation or the strong binding of graphene flakes to the hydrocarbon based matrix providing for such an environment. The percolation threshold in thermal composites was predominately discussed in the context of carbon nanotubes and carbon fibers [130-133]. Results from these cylindrical high-aspect ratio fillers are not easily extendable to geometrically disparate graphene fillers. The physics of thermal percolation is debatable in these composite systems [25,130-133]. The thermal percolation threshold can be less pronounced than the electrical percolation threshold due to heat conduction by the matrix. With the addition of graphene to the graphene-PCM composites, the lack of change in electrical conductivity was notably observed. This fact coupled with prior work in thermal interface materials and graphene fillers [26,30], make it reasonable to assume that evenly dispersed graphene flakes are unlikely to form an electrically percolating network at 1 wt. \%. The second possible reason is good thermal coupling between the fillers and matrix. The increase of the thermal conductivity in these composites can be explained by optimal attachment of the graphene flakes to the hydrocarbon molecular chains during synthesis at a prescribed processing temperature. The heat transport can go partially via the graphene fillers and partially via the matrix. Graphene has a much lower thermal Kapitza resistance, $R_{B}=\Delta T /(Q / A)$ (where $\Delta T$ is temperature difference between two materials forming an interface, $Q$ is the heat flux, and $A$ is the surface area), to several matrix materials as compared with carbon nanotubes (CNTs) to the same [1,30]. CNTs do not couple well to the matrix material or contact surface, which results in high thermal boundary resistance (TBR) between CNT filler and matrix materials and in large values of $R_{B}$. The large TBR at the CNT matrix interface can be attributed to the fundamental property-high Kapitza resistance [134] between 
one-dimensional CNTs and bulk material owing to the large difference in the phonon density of states. The attachment need not be covalent for improved heat conduction between fillers and matrix [133].

Figure 4. Thermal properties of hybrid graphene-PCM. (a) Enhancement factor of thermal conductivity of the graphene-paraffin composites with different graphene loading as a function of temperature. The results for pristine paraffin are also shown for comparison; (b) Specific heat of the composites and reference pristine paraffin as a function of temperature. The image is reprinted with permission from [29]. Copyright 2014 Elsevier.
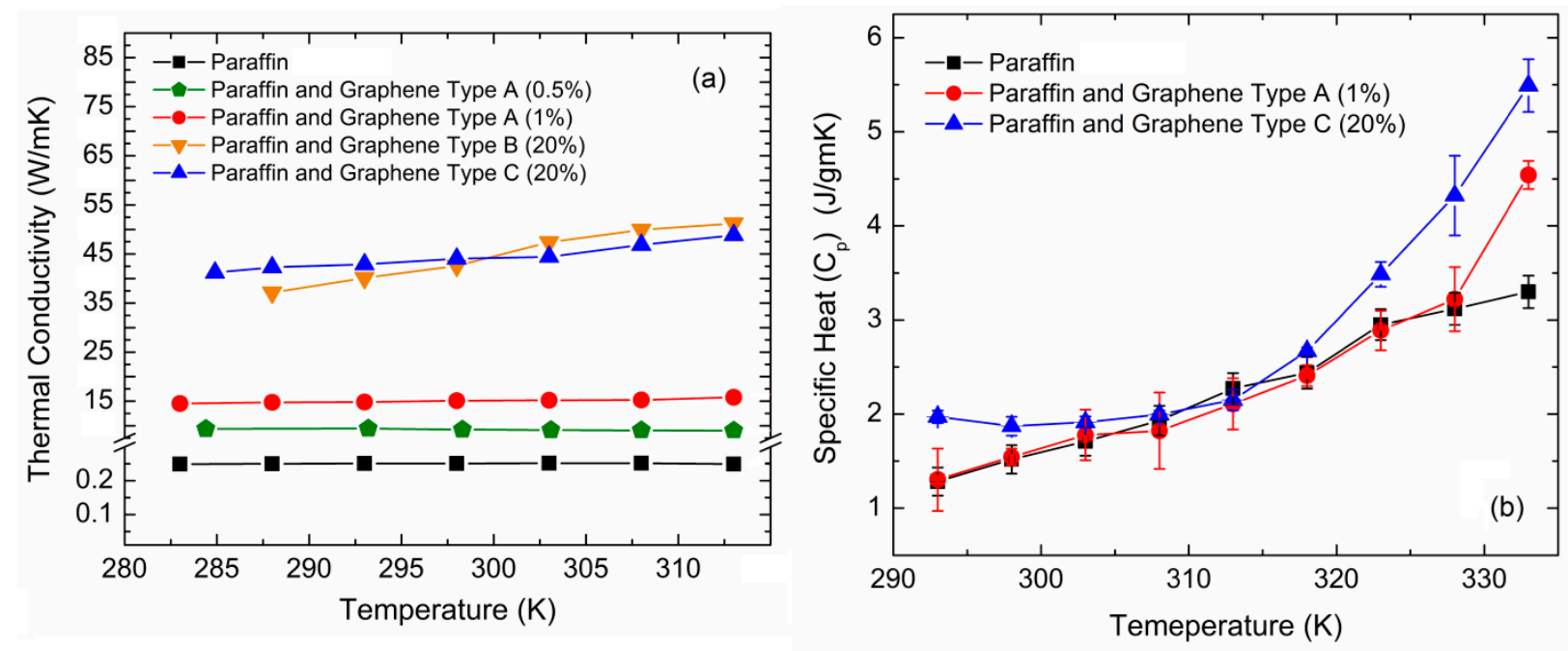

Thermal coupling in the case between graphene fillers and paraffin matrix is likely to be stronger than other matrix-filler combinations. The density function theory (DFT) calculations and molecular dynamics (MD) simulations indicated a possibility of strong increase of the thermal conductivity in ordered graphene composites with organic matrix where the heat transport is along the direction of the graphene atomic planes. Enhancements of up to $K / K_{m} \approx 360$ have been reported for a graphene loading of 5\% [135]. The exceptionally strong anisotropic increase in $K$ was attributed to graphene's planar geometry and good coupling to the octane molecules [135-137]. The heat carrying phonon modes excited in graphene coupled well to those in the organic molecules of the matrix material. Although a direct comparison between the experimental data for the graphene-paraffin composite and the composite studied in Ref. [135] theoretically is not possible, one can conclude that even randomly oriented graphene flakes should produce a strong increase in the thermal conductivity of composites. The dependence of thermal conductivity to a wide temperature range is relatively weak in all hybrid graphene-PCM composites, providing additional benefits for practical PCM-based applications. The opportunities for improvement in such thermal management applications can be obtained only if increasing thermal conductivity will not degrade the inherent latent heat storage properties of PCMs. Figure $4 \mathrm{~b}$ presents the specific heat data in the examined temperature range. The specific heat for the reference paraffin wax is $\sim 2 \mathrm{~kJ} / \mathrm{kgK}$ at RT. The addition of graphene as filler in the paraffin matrix does not significantly change the specific heat near RT. As temperature increases some increase in $C_{p}$ is likewise apparent, but this is expected as the temperature increases above $320-330 \mathrm{~K}$. One should note that the experimental uncertainty increases substantially near the melting point. 


\section{Application of Graphene-Enhanced PCMs in Battery Packs}

In this section we give an example of a specific practical application using graphene-enhanced PCMs as energy storage for thermal management in battery packs [29]. The goal in this application was to increase PCM's thermal conductivity without degrading its latent heat storage ability. The battery packs consisted of cylindrical Li-ion batteries connected to a charging-discharging setup that delivered continuous charging-discharging cycles of 16A and 5A, respectively. During the pre-set ten charge-discharge cycles, temperature measurements were logged at assigned time intervals using strategically placed thermocouples and a data acquisition system (DAS). Two thermocouples were attached to the cathode and anode ends of a battery cylinder inside of the battery pack; a third thermocouple was attached to the battery pack shell that was acting as the heat sink; and a fourth thermocouple exposed to the ambient environment. The battery packs themselves were prepared with different media, including air (i.e., no PCM), pristine paraffin PCM, and hybrid graphene-PCM with different graphene loading fractions. The paraffin PCM used in these battery packs was melted, mixed with graphene solutions (as applicable), and allowed to cool to RT before testing. All experiments followed the same test and setup protocols. The representative results of the tests of the hybrid graphene-PCM reported by Balandin and co-workers [29] are presented in Figure 5.

Figure 5. (a) Optical image of battery pack with six cylindrical Li-ion batteries. The space among the batteries is filled with paraffin PCM or graphene-enhanced PCM for testing under real-life conditions; (b) Diagram of the temperature rise inside the Li-ion battery pack during the first ten charging - discharging cycles for the battery pack without PCM (red), with conventional paraffin PCM (blue), with the hybrid graphene-PCM at 1 wt. \% loading (orange) and with the hybrid graphene-PCM at $20 \mathrm{wt}$ \% loading (green). Further reduction in the temperature rise is possible through a more robust design of the outside heat sink. The image in the right panel is reprinted with permission from [29]. Copyright 2014 Elsevier.

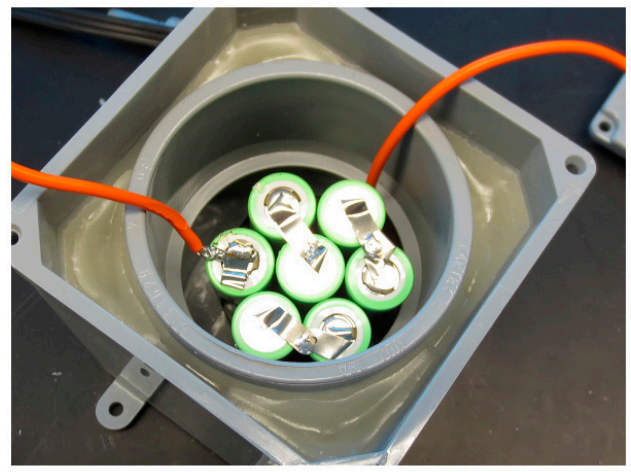

(a)

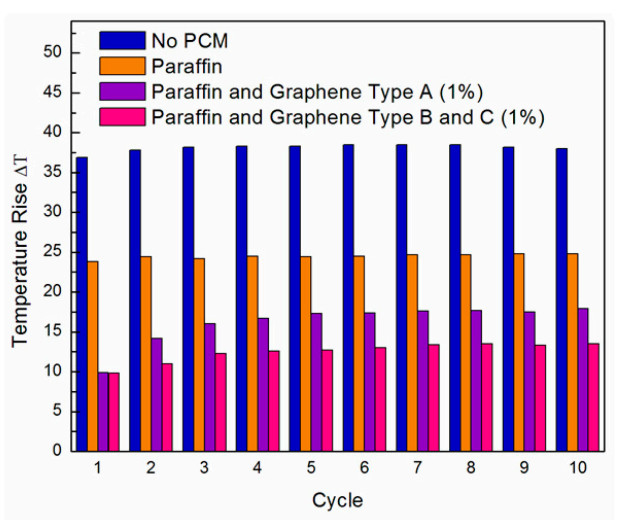

(b)

The empirical results from four different battery pack design configurations are presented in Figure 5. In the battery pack configuration where no PCM is used heat is dissipated via the ambient air and the metal bottom of the pack. This configuration produces the highest temperature rise at $\Delta T \sim 37{ }^{\circ} \mathrm{C}$ inside the battery to the ambient environment as measured by the thermocouples attached to 
the anode/cathode ends. Adding conventional paraffin PCM as the medium in the second battery pack configuration decreases the temperature rise to $\sim 24{ }^{\circ} \mathrm{C}$. Further decrease in the temperature rise is realized with graphene-enhanced PCM as the medium in these Li-ion battery packs as indicated in the third battery pack configuration. The lowest temperature rise of $\sim 10{ }^{\circ} \mathrm{C}$ was measured during the first charge-discharge cycle which then stabilizes to $\sim 16{ }^{\circ} \mathrm{C}$ after the third cycle and beyond. Improvement is observed further at a saturation temperature rise of $\sim 13{ }^{\circ} \mathrm{C}$ in the fourth battery pack configuration with a higher graphene loading fraction of the hybrid graphene-PCM. The behavior observed in the dependence of $\Delta T$ to the number of cycles, seen in Figure 5, reflects the physical cooling mechanism characteristic of conventional PCMs and hybrid graphene-PCMs. The conventional PCM medium absorbs and stores most of the heat, while conducting a small amount to the outer battery pack shell. For the hybrid graphene-PCM medium the storage and conduction of heat occurs simultaneously and in the aforementioned configurations inhibited the PCM from reaching its melting point of $70{ }^{\circ} \mathrm{C}$. The graphene-enhanced PCM medium results in a lower temperature rise inside the battery pack, but also increases the temperature of the outside shell. These conditions are ideal for practical applications in the automotive and aerospace industries where an appropriately designed thermally dissipating path from battery packs to heat sinks, e.g. vehicle frame, would eliminate or reduce the temperature rise of the outside shell, further improving thermal management.

\section{Modeling-Based Optimization of Thermal Management with Graphene-Enhanced PCMs}

Computer simulation of thermal management systems for battery packs provides valuable information for materials and system optimization. We corroborated our experimental results with the numerical solution defined by the heat diffusion equation for transient conductive heat transfer in solids and parametric data of the empirical battery design. These parameters along with the measured specific heat and thermal conductivity properties of the synthesized composites were used to model the conductive heat flow in the Li-ion battery pack using COMSOL's computer simulation software package. The constructed three-dimensional (3D) model facilitated the analysis of the six-cylinder battery pack in different media. The six batteries, $18.4 \mathrm{~mm}$ in diameter, were evenly distributed within a medium represented as a solid cylinder, $70 \mathrm{~mm}$ in diameter, all of which were enclosed within a $1 \mathrm{~mm}$ thick aluminum sheath. Due to the battery pack's simple construction, an extra coarse mesh of free tetrahedrals was used for the solid cylinders, heat conductive medium within which the solid cylinders were encased, and aluminum sheath. The transient conductive heat transfer equation was solved to determine the temperature rise inside and outside of the battery pack [29]. In all simulation runs only the material characteristics of the medium that fill the space among the battery cylinders were modified. The different media included air, conventional paraffin PCM, and graphene-enhanced PCM. For conventional paraffin PCM without graphene we used thermal conductivity $K=0.25 \mathrm{~W} / \mathrm{mK}$, mass density $900 \mathrm{~kg} / \mathrm{m}^{3}$, and heat capacity $2500 \mathrm{~J} / \mathrm{kgK}$. The simulation results included a transient analysis of temperature $v s$. time for any specific point inside the 3D modeled battery pack at any given time. Specific locations of the data analyzed correspond to the physical placement of thermocouples in the empirical test setup. Empirical data from the thermocouples were compared to these simulation results.

Figure 6 illustrates the Li-ion battery pack used in both empirical test setups and modeling simulations. Included are the simulated temperature profiles of four different cases that correspond to 
actual experimental setups. In the case of air (i.e., no PCM) serving as the medium between battery cylinders, the cylinders reach their maximum temperature at $333 \mathrm{~K}$. Upon introduction of conventional paraffin PCM as the medium the maximum temperature of the cylinders drops to $320 \mathrm{~K}$. However, the best performing thermal management of these battery cylinders comes from the use of hybrid graphene-PCM medium with maximum temperatures of $310-315 \mathrm{~K}$ resulting also in a more uniform temperature profile. One should note here that substantial reduction of the temperature of the battery cylinders is achieved even at small loading fractions of graphene.

Figure 6. Schematic of the battery pack and simulated temperature rise. The structure parameters correspond to the experimentally tested batteries. Modeling based optimization of the materials and pack parameters are essential for practical applications of graphene-enhanced PCMs in energy storage technologies.

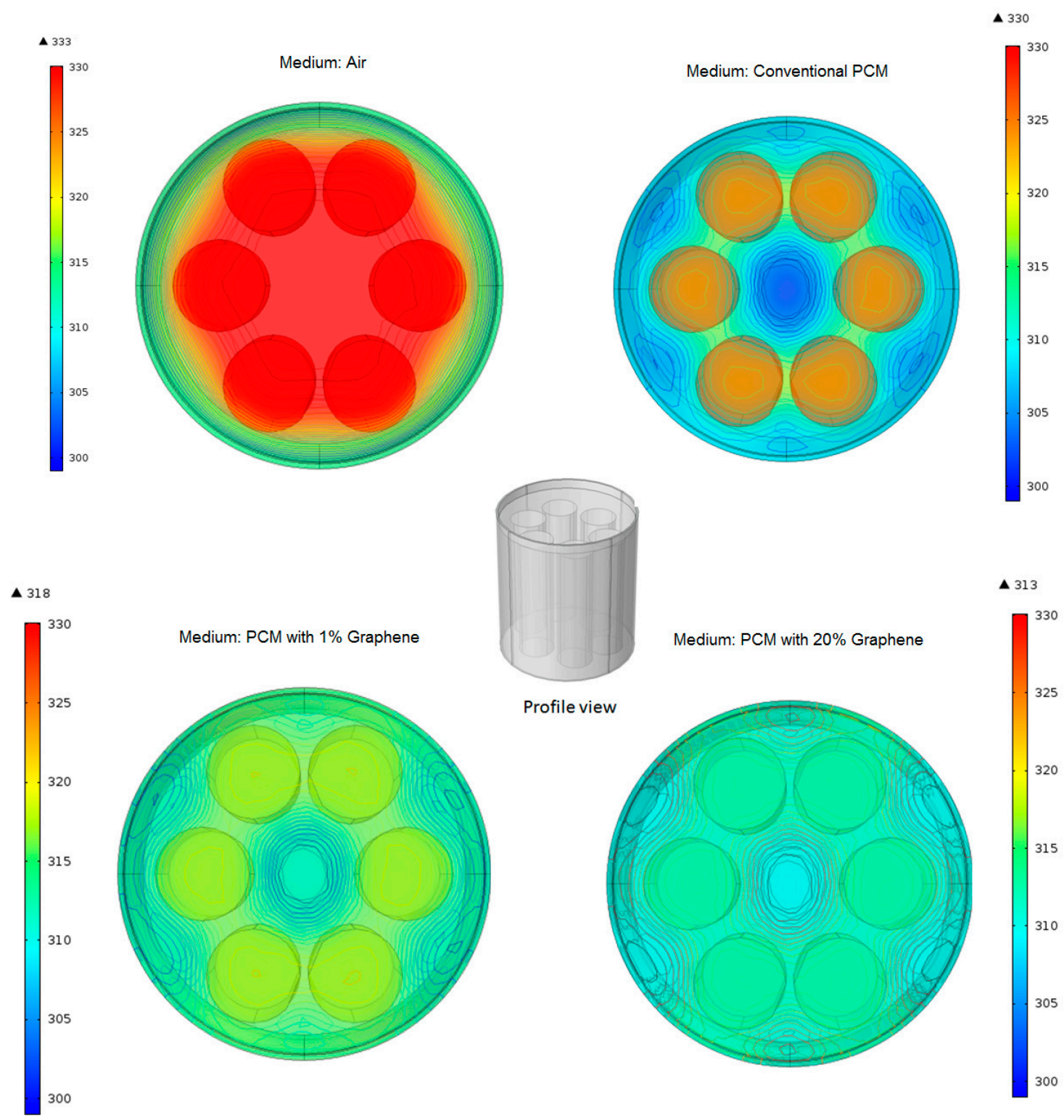




\section{Conclusions}

In this paper we presented a review of the thermal properties of graphene and few-layer graphene. The results of the experimental and theoretical studies of thermal conductivity at room temperature and above are summarized in a comprehensive table. The practical applications of graphene in thermal management are outlined in an example using thermal phase change materials. It is shown that the use of liquid-phase-exfoliated graphene as filler material in phase change materials is promising for thermal management of high-power battery packs. The described results indicate that graphene has the potential to outperform metal nanoparticles and carbon allotropes as filler in materials for thermal management.

\section{Acknowledgments}

The work at UC Riverside was supported, in part, by the Center for Function Accelerated nanoMaterial Engineering (FAME). FAME Center is one of six centers of STARnet-A Semiconductor Research Corporation (SRC) program sponsored by MARCO and DARPA and the National Science Foundation (NSF) project ECCS 1307671. D.L.N. acknowledges the financial support from the Republic of Moldova through the projects $11.817 .05 .10 \mathrm{~F}$ and $14.819 .16 .02 \mathrm{~F}$ and from the Science and Technology Center in Ukraine (STCU, project \#5937). J.D.R.'s work was funded in part by the National Science Foundation (NSF) SBIR project.

\section{Author Contributions}

A.A.B. envisioned and coordinated the invited review paper preparation; D.L.N. wrote the theoretical analysis part of the review and contributed to manuscript preparation; J.D.R. participated in the investigation of the graphene enhanced thermal phase change materials, performed numerical simulations and contributed to manuscript preparation.

\section{Conflicts of Interest}

The authors declare no conflict of interest.

\section{References}

1. Balandin, A.A. Thermal properties of graphene and nanostructured carbon materials. Nat. Mater. 2011, 10, 569-581.

2. Nika, D.L.; Balandin, A.A. Two-dimensional phonon transport in graphene. J. Phys. 2012, 24, 233203.

3. Balandin, A.A.; Nika, D.L. Phonons in low-dimensions: Engineering phonons in nanostructures and grapheme. Mater. Today 2012, 15, 266-275.

4. Balandin, A.A.; Ghosh, S.; Bao, W.; Calizo, I.; Teweldebrhan, D.; Miao, F.; Lau, C.N. Superior thermal conductivity of single layer grapheme. Nano Lett. 2008, 8, 902-907.

5. Ghosh, S.; Calizo, I.; Teweldebrhan, D.; Pokatilov, E.P.; Nika, D.L.; Balandin, A.A.; Bao, W.; Miao, F.; Lau, C.N. Extremely high thermal conductivity in graphene: Prospects for thermal management application in nanoelectronic circuits. Appl. Phys. Lett. 2008, 92, 151911. 
6. Ferrari, A.C.; Meyer, J.C.; Scardaci, V.; Casiraghi, C.; Lazzeri, M.; Mauri, F.; Piscanec, S.; Jiang, D.; Novoselov, K.S.; Roth, S.; et al. Raman Spectrum of Graphene and Graphene Layers. Phys. Rev. Lett. 2006, 97, 187401.

7. Calizo, I.; Balandin, A.A.; Bao, W.; Miao, F.; Lau, C.N. Temperature Dependence of the Raman Spectra of Graphene and Graphene Multilayers. Nano Lett. 2007, 7, 2645-2649.

8. Calizo, I.; Miao, F.; Bao, W.; Lau, C.N.; Balandin, A.A. Variable temperature Raman microscopy as a nanometrology tool for graphene layers and graphene-based devices. Appl. Phys. Lett. 2007, 91, 071913.

9. Calizo, I.; Bao, W.; Miao, F.; Lau, C.N.; Balandin, A.A. The effect of substrates on the Raman spectrum of graphene: Graphene-on-sapphire and graphene-on-glass. Appl. Phys. Lett. 2007, 91, 201904.

10. Calizo, I.; Bejenari, I.; Rahman, M.; Liu, G.; Balandin, A.A. Ultraviolet Raman microscopy of single and multilayer grapheme. J. Appl. Phys. 2009, 106, 043509.

11. Teweldebrhan, D.; Balandin, A.A. Modification of graphene properties due to electron-beam irradiation. Appl. Phys. Lett. 2009, 94, 013101.

12. Sadeghi, M.M.; Pettes, M.T.; Shi, L. Thermal transport in graphene. Solid State Commun. 2012, 152, 1321-1330.

13. Wemhoff, A.P. A review of theoretical techniques for graphene and graphene nanoribbons thermal conductivity prediction. Int. J. Transp. Phenom. 2012, 13, 121.

14. Balandin, A. Better computing through CPU cooling. IEEE Spectr. 2009, 29, 35-39.

15. Garimella, S.V.; Fleischer, A.S.; Murthy, J.Y.; Keshavarzi, A.; Prasher, R.; Patel, C.; Bhavnani, S.H.; Venkatasubramanian, R.; Mahajan, R.; Joshi, Y.; et al. Thermal Challenges in Next-Generation Electronic Systems. IEEE Trans. Compon. Packag. Technol. 2008, 31, 801-815.

16. Linden, D.; Reddy, B.T. Handbook of Batteries; McGraw-Hill: New York, NY, USA, 2002.

17. Spotnitz, R.; Franklin, J. Abuse behavior of high-power, lithium-ion cells. J. Power Sources 2003, 113, 81-100.

18. Mikolajczak, C.; Kahn, M.; White, K.; Long, R.T. Lithium-Ion Batteries Hazard and Use Assessment. Fire Prot. Res. Found. 2011, 76, 102.

19. FAA Press Release. Federal Aviation Administration; FAA Press: Washington, DC, USA, 2013.

20. Zalba, B.; Marin, J.M.; Cabeza, L.F. Review on thermal energy storage with phase change: Materials, heat transfer analysis and applications. Appl. Ther. Eng. 2003, 23, 251-283.

21. Agyenim, F.; Hewitt, N.; Eames, P.; Mervyn, S. A review of materials, heat transfer and phase change problem formulation for latent heat thermal energy storage systems (LHTESS). Renew Sustain. Energy Rev. 2010, 14, 615-628.

22. Farid, M.M.; Khudhair, A.M.; Razack, S.A.; Al-Hallaj, S. A review on phase change energy storage: Materials and applications. Energy Convers. Manag. 2004, 45, 1597-1615.

23. Sharma, S.D.; Sagara, K. Latent heat storage materials and systems: A review. J. Green Energy 2005, 2, 1-56.

24. Yu, A.; Ramesh, P.; Itkis, M.E.; Bekyarova, E.; Haddon, R.C. Graphite nanoplatelet-epoxy composite thermal interface materials. J. Phys. Chem. C 2007, 111, 7565-7569.

25. Prasher, R. Thermal conductivity of composites of aligned nanoscale and microscale wires and pores. Proc. IEEE 2006, 94, 1571-1585. 
26. Shahil, K.M.F.; Balandin, A.A. Graphene-Multilayer graphene nanocomposites as highly efficient thermal interface materials. Nano Lett. 2012, 12, 861-867.

27. Yan, A.; Liu, G.; Khan, J.M.; Balandin, A.A. Graphene quilts for thermal management of high-power GaN transistors. Nat. Commun. 2012, 3, 827.

28. Goyal, V.; Balandin, A.A. Thermal properties of the hybrid graphene-metal nano-micro-composites: Applications in thermal interface materials. Appl. Phys. Lett. 2012, 100, 073113.

29. Goli, P.; Legedza, S.; Dhar, S.; Salgado, R.; Renteria, J.; Balanidn, A.A. Graphene-enhanced hybrid phase change materials for thermal management of Li-ion batteries. J. Power Sources 2014, 248, 37-43.

30. Shahil, K.M.F.; Balandin, A.A. Thermal properties of graphene and multilayer graphene: Applications in thermal interface materials. Solid State Commun. 2012, 152, 1331-1340.

31. Ghosh, S.; Bao, W.; Nika, D.L.; Subrina, S.; Pokatilov, E.P.; Lau, C.N.; Balandin, A.A. Dimensional crossover of thermal transport in few-layer graphene. Nat. Mater. 2010, 9, 555-558.

32. Cai, W.; Moore, A.L.; Zhu, Y.; Li, X.; Chen, S.; Shi, L.; Ruoff, R.S. Thermal transport in suspended and supported monolayer graphene grown by chemical vapor deposition. Nano Lett. 2010, 10, 1645-1651.

33. Jauregui, L.A.; Yue, Y.; Sidorov, A.N.; Hu, J.; Yu, Q.; Lopez, G.; Jalilian, R.; Benjamin, D.K.; Delk, D.A.; Wu, W.; et al. Thermal transport in graphene nanostructures: Experiments and simulations. ECS Trans. 2010, 28, 73-83.

34. Mak, K.F.; Shan, J.; Heinz, T.F. Seeing many-body effects in single and few layer graphene: Observation of two-dimensional saddle point excitons. Phys. Rev. Lett. 2011, 106, 046401.

35. Kim, K.S.; Zhao, Y.; Jang, H.; Lee, S.Y.; Kim, J.M.; Kim, K.S.; Ahn, J.; Kim, P.; Choi, J.; Hong, B.H. Large-scale pattern growth of graphene films for stretchable transparent electrodes. Nature 2009, 457, 706-710.

36. Kravets, V.G.; Grigorenko, A.N.; Nair, R.R.; Blake, P.; Anissimova, S.; Novoselov, K.S.; Geim, A.K. Spectroscopic ellipsometry of graphene and an exciton-shifted van Hove peak in absorption. Phys. Rev. B 2010, 81, 155413.

37. Seol, J.H.; Jo, I.; Moore, A.L.; Lindsay, L.; Aitken, Z.H.; Pettes, M.T.; Li, X.; Yao, Z.; Huang, R.; Broido, D.; et al. Two-dimensional phonon transport in supported graphene. Science 2010, 328, 213-216.

38. Maultzsch, J.; Reich, S.; Thomsen, C.; Requardt, H.; Ordejon, P. Phonon dispersion in graphite. Phys. Rev. Lett. 2004, 92, 075501.

39. Mounet, N.; Marzari, N. First-principles determination of the structural, vibrational and thermodynamic properties of diamond, graphite, and derivatives. Phys. Rev. B 2005, 71, 205214.

40. Wirtz, L.; Rubio, A. The phonon dispersion of graphite revisited. Solid State Commun. 2004, 131, 141-152.

41. Nika, D.L.; Pokatilov, E.P.; Askerov, A.S.; Balandin, A.A. Phonon thermal conduction in graphene: Role of Umklapp and edge roughness scattering. Phys. Rev. B 2009, 79, 155413.

42. Nika, D.L.; Ghosh, S.; Pokatilov, E.P.; Balandin, A.A. Lattice thermal conductivity of graphene flakes: Comparison with bulk graphite. Appl. Phys. Lett. 2009, 94, 203103.

43. Nika, D.L.; Askerov, A.S.; Balandin, A.A. Anomalous size dependence of the thermal conductivity of graphene ribbons. Nano Lett. 2012, 12, 3238-3244. 
44. Cocemasov, A.I.; Nika, D.L.; Balandin, A.A. Phonons in twisted bilayer graphene. Phys. Rev. B 2013, 88, 035428.

45. Falkovsky, L.A. Symmetry constraints on phonon dispersion in graphene. Phys. Lett. A 2008, 372, 5189-5192.

46. Perebeinos, V.; Tersoff, J. Valence force model for phonons in graphene and carbon nanotubes. Phys. Rev. B 2009, 79, 241409.

47. Droth, M.; Burkard, G. Acoustic phonon and spin relaxation in graphene nanoribbons. Phys. Rev. $B$ 2011, 84, 155404.

48. Qian, J.; Allen, M.J.; Yang, Y.; Dutta, M.; Stroscio, M.A. Quantized long-wavelength optical phonon modes in graphene nanoribbon in the elastic continuum model. Superlattices Microstruct. 2009, 46, 881-888.

49. Alofi, A.; Srivastva, G.P. Phonon conductivity in graphene. J. Appl. Phys. 2012, 112, 013517.

50. Yan, J.-A.; Ruan, W.Y.; Chou, M.Y. Phonon dispersions and vibrational properties of monolayer, bilayer, and trilayer graphene: Density-functional perturbation theory. Phys. Rev. B 2008, $77,125401$.

51. Dubay, O.; Kresse, G. Accurate density functional calculations for the phonon dispersion relation of graphite layer and carbon nanotubes. Phys. Rev. B 2003, 67, 035401.

52. Wang, H.; Wang, Y.; Cao, X.; Feng, M.; Lan, G. Vibrational properties of graphene and graphene layers. J. Raman Spectrosc. 2009, 40, 1791-1796.

53. Lindsay, L.; Broido, D. Optimized Tersoff and Brenner empirical potential parameters for lattice dynamics and phonon thermal transport in carbon nanotubes and graphene. Phys. Rev. B 2010, 81, 205441.

54. Lindsay, L.; Broido, D.A.; Mingo, N. Flexural phonons and thermal transport in multilayer graphene and graphite. Phys. Rev. B 2011, 83, 235428.

55. Singh, D.; Murthy, J.Y.; Fisher, T.S. Mechanism of thermal conductivity reduction in few-layer graphene. J. Appl. Phys. 2011, 110, 044317.

56. Zhong, W.R.; Zhang, M.P.; Ai, B.Q.; Zheng, D.Q. Chirality and thickness-dependent thermal conductivity of few-layer graphene: A molecular dynamics study. Appl. Phys. Lett. 2011, 98, 113107.

57. Evans, W.J.; Hu, L.; Keblinsky, P. Thermal conductivity of graphene ribbons from equilibrium molecular dynamics: Effect of ribbon width, edge roughness, and hydrogen termination. Appl. Phys. Lett. 2010, 96, 203112.

58. Zhang, H.; Lee, G.; Cho, K. Thermal transport in graphene and effects of vacancies. Phys. Rev. $B$ 2011, 84, 115460 .

59. Wei, Z.; Ni, Z.; Bi, K.; Chen, M.; Chen, Y. In-plane lattice thermal conductivities of multilayer graphene films. Carbon 2011, 49, 2653-2658.

60. Ong, Z.-Y.; Pop, E. Effect of substrate modes on thermal transport in supported graphene. Phys. Rev. B 2011, 84, 075471.

61. Wei, N.; Xu, L.; Wang, H.-Q.; Zheng, J.-C. Strain engineering of thermal conductivity in graphene sheets and nanoribbons: A demonstration of magic flexibility. Nanotechnology 2011, 22,105705 .

62. Hao, F.; Fang, D.; Xu, Z. Mechanical and thermal transport properties of graphene with defects. Appl. Phys. Lett. 2011, 99, 041901. 
63. Mortazavi, B.; Ahzi, S. Thermal conductivity and tensile response of defective graphene: A molecular dynamics study. Carbon 2013, 63, 460-470.

64. Ng, T.; Yeo, J.J.; Liu, Z.S. A molecular dynamics study of the thermal conductivity of graphene nanoribbons containing dispersed Stone-Thrower-Wales defects. Carbon 2012, 50, 4887-4893.

65. Jang, Y.Y.; Cheng, Y.; Pei, Q.X.; Wang, C.W.; Xiang, Y. Thermal conductivity of defected graphene. Phys. Lett. A 2012, 376, 3668-3672.

66. Yeo, J.J.; Liu, Z.; Ng, T.Y. Comparing the effects of dispersed Stone-Thrower-Wales defects and doule vacancies on the thermal conductivity of graphene nanoribbons. Nanotechnology 2012, 23, 385702.

67. Yang, D.; Ma, F.; Sun, Y.; Hu, T.; Xu, K. Influence of typical defects on thermal conductivity of graphene nanoribbons: An equilibrium molecular dynamics simulation. Appl. Surf. Sci. 2012, 258, 9926-9931.

68. Park, M.; Lee, S.C.; Kim, Y.S. Length-dependent thermal conductivity of graphene and its macroscopic limit. J. Appl. Phys. 2013, 114, 053506.

69. Yu, C.; Zhang, G. Impacts of length and geometry deformation on thermal conductivity of graphene nanoribbons. J. Appl. Phys. 2013, 113, 044306.

70. Cao, A. Molecular dynamics simulation study on heat transport in monolayer graphene sheet with various geometries. J. Appl. Phys. 2012, 111, 083528.

71. Cao, H.-Y.; Guo, Z.-X.; Xiang, H.; Gong, Z.-G. Layer and size dependence of thermal conductivity in multilayer graphene. Phys. Lett. A 2012, 373, 525-528.

72. Cheng, L.; Kumar, S. Thermal transport in graphene supported copper. J. Appl. Phys. 2012, 112, 043502.

73. Yeo, P.S.E.; Loh, K.P.; Gan, C.K. Strain dependence of the heat transport properties of graphene nanoribbons. Nanotechnology 2012, 23, 495702.

74. Ma, F.; Zheng, H.B.; Sun, Y.J.; Yang, D.; Xu, K.W.; Chu, P.K. Strain effect on lattice vibration, heat capacity, and thermal conductivity of graphene. Appl. Phys. Lett. 2012, 101, 111904.

75. Huang, Z.; Fisher, S.; Murthy, Y. Simulation of phonon transmission through graphene and graphene nanoribbons with a Green's function method. J. Appl. Phys. 2010, 108, 094319.

76. Zhai, X.; Jin, G. Stretching-enhanced ballistic thermal conductance in graphene Nanoribbons. Europhys. Lett. 2011, 96, 16002.

77. Klemens, P.G. Theory of the a-plane thermal conductivity of graphite. J. Wide Bandgap. Mater. 2000, 7, 332-339.

78. Klemens, P.G.; Pedraza, D.F. Thermal conductivity of graphite in basal plane. Carbon 1994, 32, $735-741$.

79. Ghosh, S.; Nika, D.L.; Pokatilov, E.P.; Balandin, A.A. Heat conduction in graphene: Experimental study and theoretical interpretation. New J. Phys. 2009, 11, 095012.

80. Nika, D.L.; Pokatilov, E.P.; Balandin, A.A. Theoretical description of thermal transport in graphene: The issues of phonon cut-off frequencies and polarization branches. Phys. Stat. Sol. B 2011, 248, 2609-2614.

81. Balandin, A.A.; Ghosh, S.; Nika, D.L.; Pokatilov, E.P. Extraordinary thermal conductivity of graphene: Ossible applications in thermal management. ECS Trans. 2010, 28, 63-71.

82. Balandin, A.A.; Ghosh, S.; Nika, D.L.; Pokatilov, E.P. Thermal conduction in suspended graphene layers. Fullerenes Nanotubes Carbon Nanostruct. 2010, 18, 474-486. 
83. Adamyan, V.; Zavalniuk, V. Lattice thermal conductivity of graphene with conventionally isotopic defects. J. Phys. 2012, 24, 415406.

84. Aksamija, Z.; Knezevic, I. Lattice thermal conductivity of graphene nanoribbons: Anisotropy and edge roughness scattering. Appl. Phys. Lett. 2011, 98, 141919.

85. Aksamija, Z.; Knezevic, I. Thermal transport in graphene nanoribbons supported on $\mathrm{SiO}_{2}$. Phys. Rev. B 2012, 86, 165426.

86. Nika, D.L.; Cocemasov, A.I.; Balandin, A.A. Specific heat of twisted graphene: Engineering phonons by atomic plane rotations. Appl. Phys. Lett. 2014, 105, 031904.

87. Savin, A.V.; Kivshar, Y.S.; Hu, B. Suppression of thermal conductivity in graphene nanoribbons with rough edges. Phys. Rev. B 2010, 82, 195422.

88. Hu, J.; Ruan, X.; Chen, Y.P. Thermal conductivity and thermal rectification in graphene nanoribbons: A molecular dynamics study. Nano Lett. 2009, 9, 2730-2735.

89. Guo, Z.; Zhang, D.; Gong, X.-G. Thermal conductivity of graphene nanoribbons. Appl. Phys. Lett. 2009, 95, 163103.

90. Chen, S.; Wu, Q.; Mishra, C.; Kang, J.; Zhang, H.; Cho, K.; Cai, W.; Balandin, A.A.; Ruoff, R.S. Thermal conductivity of isotopically modified graphene. Nat. Mater. 2012, 11, 203-207.

91. Ouyang, T.; Chen, Y.P.; Yang, K.K.; Zhong, J.X. Thermal transport of isotopic-superlattice graphene nanoribbons with zigzag edge. Europhys. Lett. 2009, 88, 28002.

92. Jiang, J.W.; Lan, J.H.; Wang, J.S.; Li, B.W. Isotopic effects on the thermal conductivity of graphene nanoribbons: Localization mechanism. J. Appl. Phys. 2010, 107, 054314.

93. Zhang, H.; Lee, G.; Fonseca, A.F.; Borders, T.L.; Cho, K. Isotope effect on the thermal conductivity of graphene. J. Nanomater. 2010, 2010, 537657.

94. Hu, J.; Schiffli, S.; Vallabhaneni, A.; Ruan, X.; Chen, Y.P. Tuning the thermal conductivity of graphene nanoribbons by edge passivation and isotope engineering: A molecular dynamics study. Appl. Phys. Lett. 2010, 97, 133107.

95. Alofi, A.; Srivastava, G.P. Thermal conductivity of graphene and graphite. Phys. Rev. B 2013, $87,115421$.

96. Lindsay, L.; Carrete, J.; Mingo, N.; Broido, D.A.; Reinecke, T.L. Phonon thermal transport in strained and unstrained graphene from first principles. Phys. Rev. B 2014, 89, 155426.

97. Serov, A.Y.; Ong, Z.-Y.; Pop, E. Effect of grain boundaries on thermal transport in graphene. Appl. Phys. Lett. 2013, 102, 033104.

98. Xu, X.; Pereira, L.F.C.; Wang, Y.; Wu, J.; Zhang, K.; Zhao, X.; Bae, S.; Bui, C.T.; Xie, R.; Thong, J.T.L.; et al. Length-dependent thermal conductivity in suspended single-layer graphene. Nat. Commun. 2014, 5, 3689.

99. Faugeras, C.; Faugeras, B.; Orlita, M.; Potemski, M.; Nair, R.R.; Geim, A.K. Thermal conductivity of graphene in Corbino membrane geometry. ACS Nano 2010, 4, 1889-1892.

100. Dorgan, V.E.; Behnam, A.; Conley, H.J.; Bolotin, K.I.; Pop, E. Hiagh-filed electric and thermal transport in suspended graphene. Nano Lett. 2013, 13, 4581-4586.

101. Jang, W.; Chen, Z.; Bao, W.; Lau, C.N.; Dames, C. Thickness-dependent thermal conductivity of encased graphene and ultrathin graphite. Nano Lett. 2010, 10, 3909-3913.

102. Wang, Z.; Xie, R.; Bui, C.T.; Liu, D.; Ni, X.; Li, B.; Thong, J.T.L. Thermal transport in suspended and supported few-layer graphene. Nano Lett. 2011, 11, 113-118. 
103. Jang, W.; Bao, W.; Ling, L.; Lau, C.N.; Dames, C. Thermal conductivity of suspended few-layer graphene by a modified T-bridge method. Appl. Phys. Lett. 2013, 103, 133102.

104. Pettes, M.T.; Jo, I.; Yao, Z.; Shi, L. Influence of polymeric residue on the thermal conductivity of suspended bilayer graphene. Nano Lett. 2011, 11, 1195-1200.

105. Murali, R.; Yang, Y.; Brenner, K.; Beck, T.; Meindl, J.D. Breakdown current density of graphene nanoribbons. Appl. Phys. Lett. 2009, 94, 243114.

106. Liao, A.D.; Wu, J.Z.; Wang, X.; Tahy, K.; Jena, D.; Dai, H.; Pop, E. Thermally limited current carrying ability of graphene nanoribbons. Phys. Rev. Lett. 2011, 106, 256801.

107. Lindsay, L.; Broido, D.A.; Mingo, N. Diameter dependence of carbon nanotube thermal conductivity and extension to the graphene limit. Phys. Rev. B 2010, 82, 161402.

108. Munoz, E.; Lu, J.; Yakobson, B.I. Ballistic thermal conductance of graphene ribbons. Nano Lett. 2010, 10, 1652-1656.

109. Jiang, J.-W.; Wang, J.-S.; Li, B. Thermal conductance of graphite and dimerite. Phys. Rev. B 2009, 79, 205418.

110. Freund, M.; Csikos, R.; Keszthelyi, S.; Mozes, G.Y. Paraffin Products Properties, Technologies, Applications; Akademiai Kiado: Budapest, Hungary, 1981; Volume 14.

111. Brown, J.K.; Sheppard, N.; Simpson, D.M. The Interpretation of the Infra-Red and Raman Spectra of the $n$-Paraffins. Math. Phys. Sci. 1954, 247, 35.

112. Edwards, H.G.M.; Falk, M.J.P. Fourier-transform Raman spectroscopic study of unsaturated and saturated waxes. Spectrochim. Acta Part A 1997, 53, 2685-2694.

113. Kalyanasundaram, K.; Thomas, J.K. The conformational state of surfactants in the solid state and in micellar form. A laser-excited Raman scattering study. J. Phys. Chem. 1976, 80, 1462-1473.

114. MacPhail, R.A.; Strauss, H.L.; Snyder, R.G. Carbon-hydrogen stretching modes and the structure of $n$-alkyl chains. 2. Long, all-trans chains. J. Phys. Chem. 1984, 88, 334-341.

115. Zheng, M.; Du, W. Phase behavior, conformations, thermodynamic properties, and molecular motion of multicomponent paraffin waxes: A Raman spectroscopy study. Vib. Spectrosc. 2006, 40, 219-224.

116. Zheng, R.; Gao, J.; Wang, J.; Chen, G. Reversible temperature regulation of electrical and thermal conductivity using liquid solid phase transitions. Nat. Commun. 2011, 2, 289.

117. Sanusi, O.; Warzoha, R.; Fleischer, A.S. Energy storage and solidificaiton of paraffin phase change material embedded with graphite nanofibers. Int. J. Heat Mass Transfer. 2011, 54, 4429-4436.

118. Schiffres, S.N.; Harish, S.; Maruyama, S.; Shiomi, J.; Malen, J.A. Tunable electrical and thermal transport in ice-templated multilayer graphene nanocomposites through freezing rate control. ACS Nano 2013, 7, 11183-11189.

119. Gustafsson, S.E. Transient plane source techniques for thermal conductivity and thermal diffusivity measurements of solid materials. Rev. Sci. Instrum. 1991, 62, 797.

120. Xia, L.; Zhang, P.; Wang, R.Z. Preparation and thermal characterization of expanded graphite/paraffin composite phase change material. Carbon 2010, 48, 2538-2548.

121. He, Y. Rapid thermal conductivity measurement with a hot disk sensor: Part 1. Theoretical considerations. Thermochim. Acta 2005, 436, 122-129.

122. He, Y. Rapid thermal conductivity measurement with a hot disk sensor: Part 2. Characterization of thermal greases. Thermochim. Acta 2005, 436, 130-134. 
123. Ghosh, S.; Teweldebrhan, D.; Morales, J.R.; Garay, J.E.; Balandin, A.A. Thermal properties of the optically transparent pore-free nanostructured yttria-stabilized zirconia. J. Appl. Phys. 2009, 106, 113507.

124. Shamsa, M.; Ghosh, S.; Calizo, I.; Ralchenko, V.; Popovich, A.; Balandin, A.A. Thermal conductivity of nitrogeneated ultrananocrystalline diamond films on silicon. J. Appl. Phys. 2008, $103,083538$.

125. Gustafsson, S.E.; Karawacki, E.; Chohan, M.A. Thermal transport studies of electrically conducting materials using the transient hot-strip technique. J. Phys. D 1986, 19, 727.

126. Gustavsson, M.; Wang, H.; Trejo, R.M.; Lara-Curzio, E.; Dinwiddie, R.B.; Gustafsson, S.E. On the Use of the Transient Hot-Strip Method for Measuring the Thermal Conductivity of High-Conducting Thin Bars. Int. J. Thermophys. 2006, 27, 1816-1825.

127. Chintakrida, K.; Weinstein, R.D.; Fleischer, A.S. A direct comparison of three different material enhancement methods on the transient thermal response of paraffin phase change material exposed to high heat fluxes. Int. J. Therm. Sci. 2011, 50, 1639-1647.

128. Ehid, R.; Weinstein, R.D.; Fleischer, A.S. The shape stabilization of paraffin phase change material to reduce graphite nanofiber settling during the phase change process. Energy Convers. Manag. 2012, 57, 60-67.

129. Wang, J.; Xie, H.; Xin, Z.; Li, Y.; Chen, L. Enhancing thermal conductivity of palmitic acid based phase change materials with carbon nanotubes as fillers. Solar Energy 2010, 84, 339-344.

130. Berhan, L.; Sastry, A.M. Modeling percolation in high-aspect-ratio fiber systems. II. The effect of waviness on the percolation onset. Phys. Rev. E 2007, 75, 041121.

131. Choi, S.U.S.; Zhang, Z.G.; Yu, W.; Lockwood, F.E.; Grulke, E.A. Anomalous thermal conductivity enhancement in nanotube suspensions. Appl. Phys. Lett. 2001, 79, 2252-2254.

132. Shenogina, N.; Shenogin, S.; Xue, L.; Keblinski, P. On the lack of thermal percolation in carbon nanotube composites. Appl. Phys. Lett. 2005, 87, 133106.

133. Gulotty, R.; Castellino, M.; Jagdale, P.; Tagliaferro, A.; Balandin, A.A. Effects of Functionalization on Thermal Properties of Single-Wall and Multi-Wall Carbon Nanotube-Polymer Nanocomposites. ACS Nano. 2013, 7, 5114-5121.

134. Kapitza, P.L. The study of heat transfer in helium II. J. Phys. USSR 1941, 4, 181.

135. Konatham, D.; Bui, K.N.D.; Papavassiliou, D.V.; Striolo, A. Simulation insights into thermally conductive graphene-based nanocomposites. Mol. Phys. 2011, 109, 97-111.

136. Konatham, D.; Striolo, A. Thermal boundary resistance at the graphene-oil interface. Appl. Phys. Lett. 2009, 95, 163105.

137. Konathan, D.; Papavassiliou, D.V.; Striolo, A. Thermal boundary resistance at the graphene-graphene interface estimated by molecular dynamics simulations. Chem. Phys. Lett. 2012, 527, 47-50.

(C) 2014 by the authors; licensee MDPI, Basel, Switzerland. This article is an open access article distributed under the terms and conditions of the Creative Commons Attribution license (http://creativecommons.org/licenses/by/4.0/). 